\title{
Accuracy of intraocular lens power calculation using three optical biometry measurement devices: the OA-2000, Lenstar-LS900 and IOLMaster-500
}

\author{
Olga Reitblat ${ }^{1} \cdot$ Adi Levy ${ }^{1}$ Guy Kleinmann ${ }^{1,2} \cdot$ Ehud I. Assia ${ }^{1,3}$
}

Received: 28 October 2017 / Revised: 2 January 2018 / Accepted: 8 February 2018 / Published online: 12 March 2018

(c) The Royal College of Ophthalmologists 2018

\begin{abstract}
Purpose To compare ocular measurements of three optical biometry devices and their application in intraocular lens (IOL) power calculations.

Methods One hundred and forty eyes which had undergone cataract extraction surgery with preoperative biometry with OA2000, IOLMaster-500, and Lenstar-LS900 were enrolled. Biometry measurements of the three devices were compared. The deviation of the postoperative refraction from the preoperative refractive target was calculated with different formulas (Barrett Universal II, Hoffer Q, Holladay I, and SRK/T). Errors in the predicted astigmatism using the Barrett toric calculator were calculated for the toric IOLs. Additional 6465 eyes in which the IOLMaster-500 failed to measure axial length (AL) were reviewed. The percentage of successful measurements using the OA-2000 in those eyes was calculated.

Results High agreement was found between the three devices for AL, anterior chamber depth, and average keratometry measurements (interclass correlation confidents: $1.000,0.970$, and 0.998 , respectively, $P<0.001$ ). The mean absolute prediction errors were similar using all formulas, ranging from 0.25 to $0.29 \mathrm{D}$, with no statistical significant difference between the three devices per each formula. The OA-2000 yielded a lower against-the-rule (ATR) centroid error in the predicted astigmatism than the IOLMaster-500 and Lenstar-LS900 $\left(0.06 \mathrm{D} \pm 0.59\right.$ at $13.4^{\circ}$ vs. $0.20 \mathrm{D} \pm 0.61$ at $14.8^{\circ}$ and $0.16 \mathrm{D} \pm 0.55$ at $21.4^{\circ}$, respectively, $P<0.001, X$-axis). Among 301 cases with unsuccessful AL readings using the IOLMaster-500, the OA-2000 had 284 (94.35\%) successful measurements.

Conclusions The OA-2000 measurements showed good agreement with those of the IOLMaster-500 and Lenstar-LS900. Our results may suggest a potential advantage of the OA-2000 device in toric IOLs calculations and AL measurement success rate.
\end{abstract}

\section{Introduction}

Precision and accuracy in biometric measurements are of outmost importance in achieving desirable refractive results following cataract extraction with an intraocular lens (IOL) implantation [1]. Optical biometry has become the gold standard for ocular measurements since the introduction of the IOLMaster (Carl Zeiss Meditec AG, Jena,

Olga Reitblat

olga.reitblat@gmail.com

Ein-Tal Eye Center, Tel-Aviv, Israel

2 Kaplan Medical Center, Rehovot, Israel affiliated with The Hebrew University, Jerusalem, Israel

3 Meir Medical Center, Kfar-Saba, Israel, affiliated with Tel-Aviv University, Ramat Aviv, Israel
Germany) in 2001 [2]. The IOLMaster is a partial coherence interferometry-based device which allows for noncontact, fast and accurate measurements. The IOLMaster500 biometer (IOLMaster) uses a $780 \mathrm{~nm}$ laser diode infrared light to measure axial length (AL). The anterior chamber depth (ACD) is measured through a lateral slit illumination. Keratometry $(K)$ readings are calculated by six measured points at a $2.5 \mathrm{~mm}$ zone on the anterior cornea.

In 2009, the Lenstar-LS900 (Lenstar) (Haag-Streit AG, Koeniz, Switzerland) was introduced [3]. Optical low coherence reflectometry technology with a $820 \mathrm{~nm}$ super luminescent diode is used in order to measure the axial ocular features, including AL, ACD, lens thickness (LT), central corneal thickness (CCT), and retinal thickness. A dual zone analysis of light-emitting diode projection at 1.65 and $2.3 \mathrm{~mm}$ of closely spaced 32 measurement points produces keratometry values. 
A new biometer, the OA-2000 (Tomey GmbH, Nagoya, Japan), uses swept coherence tomography with a laser wavelength of $1060 \mathrm{~nm}$ for measuring the AL, ACD, CCT, and LT. Simultaneously, the corneal surface is measured by Placido disc topography of nine rings at 256 reference points each in one measurement [4].

Several studies have already evaluated the agreement of ocular measurements using the OA-2000 with the IOLMaster and the Lenstar [4-6]. However, there are scant data available regarding the accuracy of IOL power calculations using these three biometers [7]. The present study aimed to evaluate the accuracy of the OA-2000 measurements, compared to the IOLMaster and Lenstar devices, using varies IOL calculation formulas, in patients following cataract extraction surgery. Our second goal was to assess its ability to perform biometry measurements in cases the IOLMaster failed.

\section{Materials and methods}

Medical records were retrospectively reviewed to identify consecutive patients who had cataract extraction by two different surgeons from January 2016 to December 2017, at a private practice clinic. The study conformed to the Declaration of Helsinki and was approved by an institutional ethics committee. Inclusion criteria were: (1) preoperative OA-2000, IOLMaster-500 and Lenstar-LS900 biometry and keratometry measurements; (2) uneventful cataract surgery; and (3) postoperative manifest refraction at least 3 weeks after surgery with a best corrected visual acuity of 6/9 or better. Exclusion criteria included any preoperative ocular comorbidity effecting visual acuity, perioperative complications, previous ocular surgery, intraoperative or postoperative complications, and incomplete data.

\section{Biometry measurements}

Each eye was evaluated during the preoperative examination with the three devices on the same day. For all instruments, the mean $\mathrm{AL}$, mean $\mathrm{ACD}$, white-to-white distance (WTW), and flat and steep $K$ values were recorded. LT measurements were taken by the OA-2000 and the Lenstar instruments. All measurements of IOLMaster and Lenstar adhered to the validation criteria described by Warren Hill (Available at: http://www.doctor-hill.com/, Accessed 5 January 2017) and according to the manufacturer's calibration guidelines for measurements with the OA-2000. The reported $K$ values were derived from the anterior corneal radii measurements using a 1.3375 keratometric index of refraction. $K$ values of the OA-2000 device were assessed over a $2.5 \mathrm{~mm}$ diameter in accordance with similar radii of curvature measurements obtained by IOLMaster and Lenstar.

All three biometric measurements were available to the surgeon and were used for IOL power calculation prior to the surgery. The implanted IOL model and power were selected according to the surgeon's preferences. All surgical procedures were done using phacoemulsification through a 2.2-2.4 mm clear corneal incisions with an in-the-bag implanted IOL.

\section{Evaluating the accuracy of IOL power calculations}

Optical biometry and keratometry readings of the OA-2000, IOLMaster, and Lenstar were used to predict the refraction of the implanted IOL with four IOL power calculation formulas. The Hoffer Q [8, 9], Holladay-1 [10], and SRK/T [11] formulas were analyzed with an excel software spreadsheet (Office Professional plus 2013, Microsoft Inc.). Calculations for the Barrett Universal II formula [12, 13] were performed using the online calculator version (Available at: http://www.apacrs.org/disclaimer.asp?info = 5. Accessed 30 December 2017). Power calculation with IOLMaster biometry for the Barrett Universal II formula was done without inserting LT values, as LT measurement is not available in this IOLMaster version. IOL power calculations were performed using the User Group for Laser Interference Biometry (ULIB, Available at: http://ocusoft. de/ulib/. Accessed 30 December 2017) IOL constants for Acrysof (Alcon Laboratories Inc.) and Tecnis (Abbott Medical Optics Inc.) IOLs, and the company's optical constants recommendation for PhysIOL (PhysIOL Inc.) IOLs.

Postoperative manifest refraction was obtained 3 weeks or later following the surgery. A prediction error for each device using the four cited formulas was calculated by subtracting the predicted refraction of each formula from the postoperative refraction. An absolute error was defined as the absolute value of the prediction error. Mean prediction errors, median absolute errors, and mean absolute errors were then calculated. The percentage of eyes within an absolute deviation from target refraction of 0.50 and 1.00 diopter (D) was calculated for each IOL power formula with the three devices.

\section{Evaluating the accuracy of astigmatism correction with toric IOLs}

Residual astigmatism for the implanted toric IOL power at the measured axis of alignment, recorded at the $\geq 3$ weeks postoperative visit, was predicted using the Barrett toric calculator (Available at: http://www.ascrs.org/barrett-toriccalculator. Accessed 30 December 2017). A surgically induced astigmatism of $0.1 \mathrm{D}$ at the corneal incision was 
assumed, as had been previously recommended by Barrett [14]. Centroid and absolute astigmatic prediction errors were calculated as the difference between the manifest refraction postoperative astigmatism adjusted to the corneal plane and the predicted residual astigmatism of the implanted IOL. Astigmatism values, defined as the difference between the steep and flat $K \mathrm{~s}$, were analyzed by vector summation, as described by Holladay et al. [15]

\section{AL measurement success rate}

To assess the OA-2000 AL measurement success rate in cases that the IOLMaster failed, an additional dataset was collected. Consecutive cases with preoperative biometry measurements, using the IOLMaster, between December 2015 and November 2017 at a private practice clinic, were reviewed. Cases with unsuccessful IOLMaster AL readings were included. In these cases, biometry measurements were performed with the OA-2000. The percentage of successful AL readings using the OA-2000 was calculated.

\section{Statistical analysis}

Power analysis was performed to determine the minimal sample size for statistical significant results. A difference of $0.125 \mathrm{D}$, half of the standard refractive interval, was considered as clinically important. A standard deviation (SD) of $0.30 \mathrm{D}$ was assumed. Based on a two-tail test, an alpha level of $5 \%$ and a power of $80 \%$, a minimal sample size of 46 eyes was required. Data were checked for normality using the Shapiro-Wilk test and normality assessment plots.

The Bland and Altman method was used to evaluate the agreement between the AL, ACD, average $K$, and WTW values, measured by the OA-2000, IOLMaster, and Lenstar devices, and LT measured by the OA-2000 and the Lenstar [16]. The 95\% limits of agreement (LoA) were provided along with Bland-Altman plots. A repeated measures ANOVA was performed for comparison. Inter-rater reliability was assessed using interclass correlation coefficient (ICC) analysis.

Double-angle plots were used to describe the differences in astigmatism measurements. Statistical analyses of astigmatism data were conducted for the double angled $X$ - and $Y$ axis components each separately.

For assessing the differences of refractive prediction errors within each formula based on measurements obtained by the OA-2000, the IOLMaster, and the Lenstar, Friedman's test was performed. The absolute astigmatic prediction errors and the vector components of each biometry measurement device were also assessed with Friedman's test. Wilcoxon Signed-Rank test was used for post hock analysis. Levene's test for the equality of variances was used to assess the distribution of the numeric prediction errors.
Statistical analysis was performed with the SPSS package (version 21.0, SPSS, Inc.) and Medcalc Statistical Software (Version 12.5.0.0 Medcalc Software, Inc.). $P<$ 0.05 was considered statistically significant. A Bonferroni correction was used for multiple comparisons.

\section{Results}

The study included 140 eyes of 90 patients comprising 57 females $(63.3 \%)$ and 33 males $(36.7 \%)$, whose mean age \pm SD was $64.2 \pm 9.8$ years (range $37-83$ years). The mean implanted IOL power was $17.64 \pm 5.15 \mathrm{D}$ (range $6-28$ ). The implanted IOL models were as follows: 35 Acrysof IOLs, 60 PhysIOL IOLs, and 45 Tecnis IOLs.

\section{Measurements compatibility}

The respective means, standard deviations, and range of the ocular parameters provided by the OA-2000, IOLMaster and Lenstar devices are shown in Table 1. High agreement was seen between the measuring devices with an ICC of: $1.000,0.970,0.998,0.948$, and 0.972 , for measurements of AL, ACD, average $K$, WTW, and LT, respectively $(P<$ 0.001). Bland-Altman plots of agreement between OA2000 against IOLMaster and Lenstar biometry devices are presented in Fig. 1. The 95\% LoA were in narrow range, as shown in Table 1 and Fig. 1.

Agreement in astigmatism measurements of the three devices was high. For the $X$ and $Y$ components, ICCs were 0.995 and 0.982 , respectively $(P<0.001)$. The IOLMaster and Lenstar showed a relative with-the-rule (WTR) deviation of 0.12 and $0.10 \mathrm{D}(P<0.001)$ compared to OA-2000 (Fig. 2, Table 1).

\section{Errors in the predicted refraction}

All three biometry devices using the various formulas showed similar accuracy within \pm 0.50 and $\pm 1.00 \mathrm{D}$ from target refraction, range: $83.6-88.6$ and $100.0 \%$ (OA-2000), 84.3-88.6 and 99.3-100.0\% (IOLMaster), and 85.0-87.9 and $100.0 \%$ (Lenstar), respectively. There was no statistically significant difference in the absolute prediction errors between the OA-2000, IOLMaster, and Lenstar per each formula (Table 2). In addition, no significant differences were found in the variances of the numeric prediction errors between the three biometry devices (Table 2).

\section{Errors in the predicted astigmatism}

A total of 78 toric IOLs were incorporated in the study. The mean $\pm \mathrm{SD}$ IOL power was $17.15 \mathrm{D} \pm 5.53$ (range 


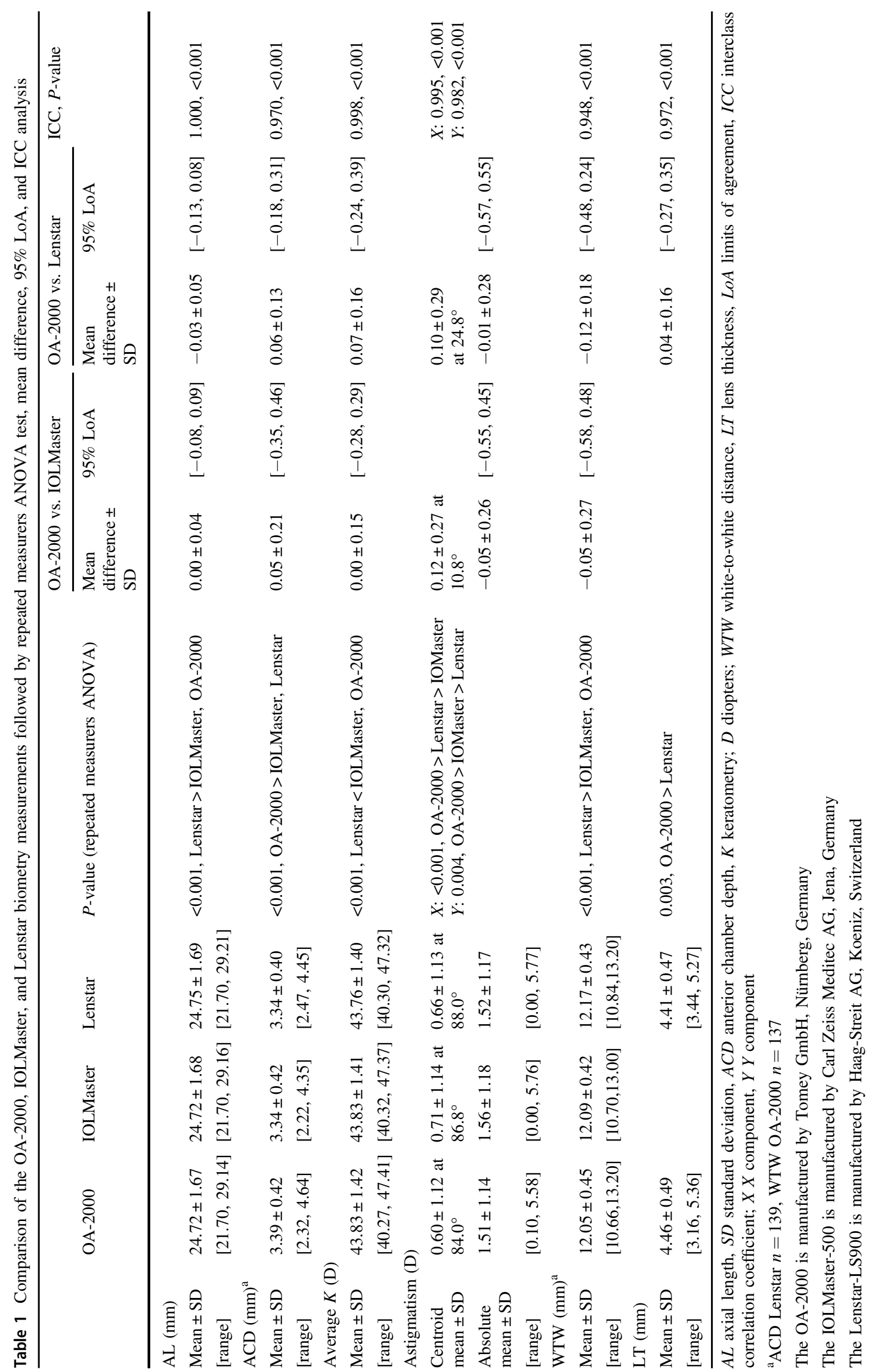


Fig. 1 Bland-Altman plots of agreement between OA-2000 (Tomey GmbH, Nagoya, Japan) against IOLMaster (Carl Zeiss Meditec, Jena, Germany) and Lenstar (Haag-Streit AG, Koeniz, Switzerland) measurements of: $\mathrm{AL} \mathrm{(a,} \mathrm{b,}$ respectively), $\mathrm{ACD}(\mathbf{c}, \mathbf{d}$, respectively), Average $K(\mathbf{e}, \mathbf{f}$, respectively), WTW (g, h, respectively), and LT (i). The solid line indicates the mean difference. The upper and lower lines represent the $95 \%$ limits of agreement. $A L$ axial length, $A C D$ anterior chamber depth, $K$ average keratometry, WTW white-to-white distance, $L T$ lens thickness
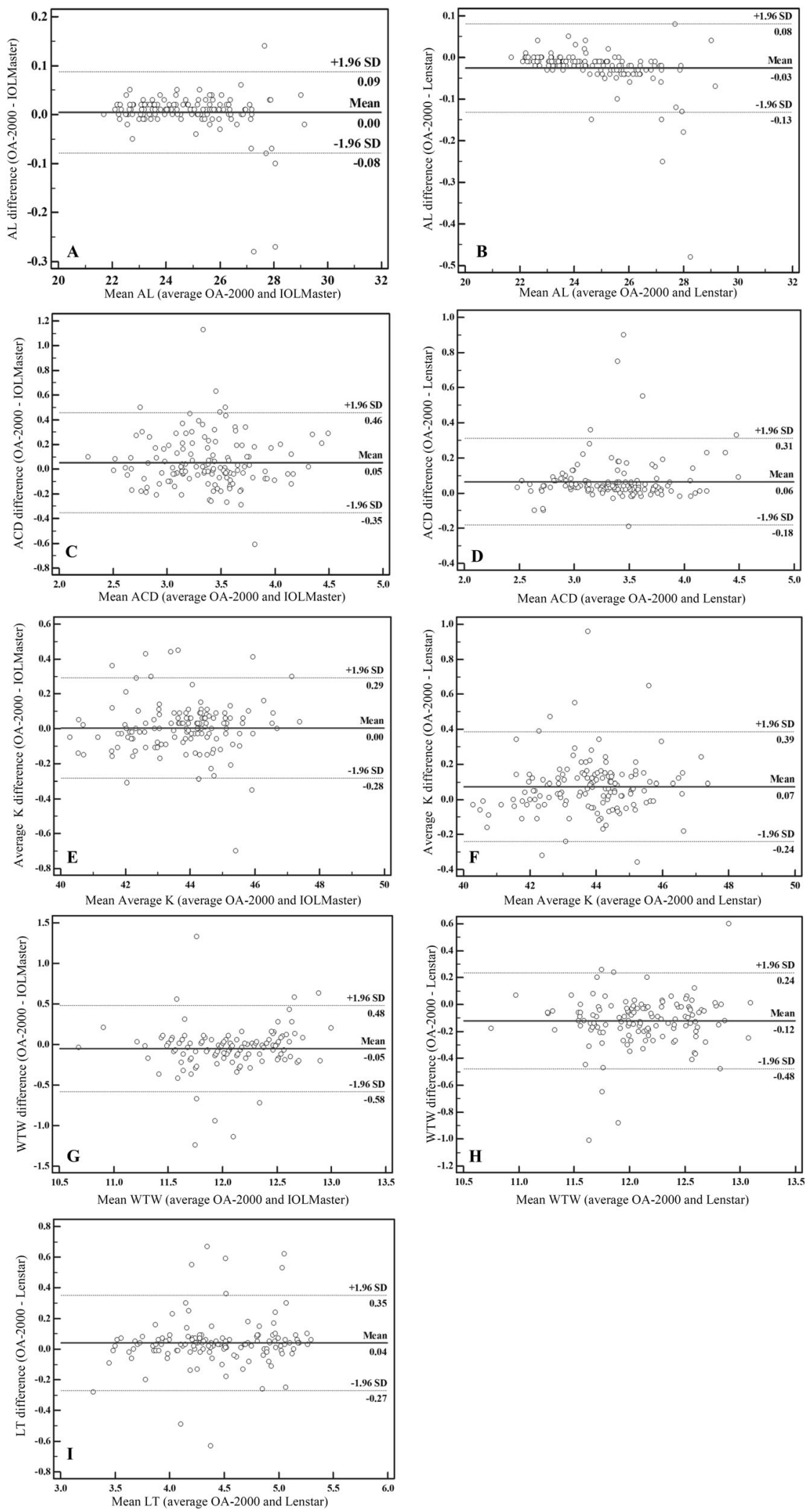

The errors in the predicted residual astigmatism for each

6.5-28.0 D). Seventy-two eyes had postoperative measurement of the implanted IOL axis and were enrolled in the toric IOLs sub-analysis. device are displayed in Table 2. The differences in the absolute error between the three measuring systems did not 
Fig. 2 Double-angle plots of the vector differences between astigmatism measurements of the OA-2000 (Tomey GmbH, Nagoya, Japan) against a IOLMaster (Carl Zeiss Meditec, Jena, Germany) and b Lenstar (Haag-Streit AG, Koeniz, Switzerland)

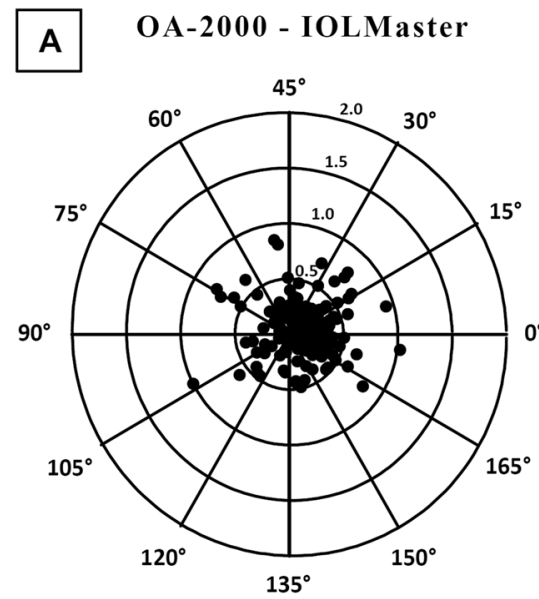

$0.12 \mathrm{D} \pm 0.27 @ 10.8^{\circ}$

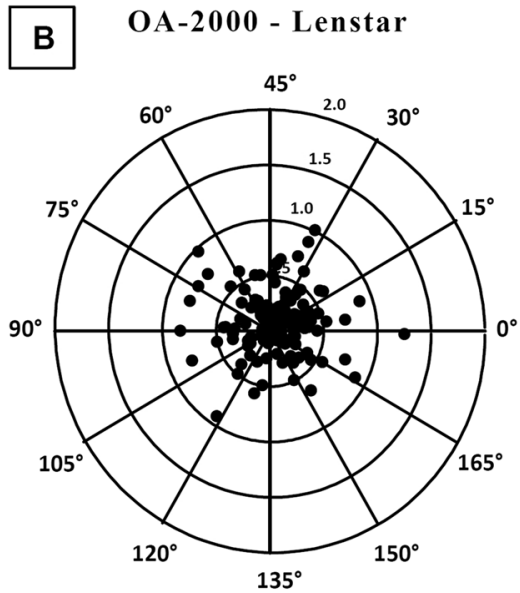

$0.10 \mathrm{D} \pm 0.29 @ 24.8^{\circ}$ reach a level of significance $(P=0.506)$. There was a small against-the-rule (ATR) centroid prediction error using all three devices in combination with the Barrett toric calculator, ranging from $0.06 \mathrm{D} \pm 0.59$ at $13.4^{\circ}(\mathrm{OA}-2000)$ to $0.20 \mathrm{D} \pm 0.61$ at $14.8^{\circ}$ (IOLMaster). The OA-2000 measurements, using the Barrett toric calculator, yielded the lowest ATR centroid prediction errors of those three devices $(P<0.001, X$-axis) (Table 2, Fig. 3).

\section{AL measurement success rate}

A total of 6465 preoperative biometry measurements using the IOLMaster were performed during the study period. In 301 eyes (4.66\%), the IOLMaster failed to measure the AL. Among those, the OA-2000 had 284 (94.35\%) successful measurements.

\section{Discussion}

This study compared the consistency and the accuracy of the results of three biometry devices: OA-2000, IOLMaster500, and Lenstar-LS900.

High agreement between the measurements provided by the three devices was found, with an ICC exceeding 0.9 for all parameters evaluated. The mean differences were small and the LoA were narrow for all comparisons.

AL obtained with the new OA-2000 biometer showed excellent agreement with the IOLMaster and the Lenstar AL measurement (ICC 1.000). The Lenstar provided a statistically significant higher AL of 0.03 compared to the OA2000. Considering a difference of $0.1 \mathrm{~mm}$ in $\mathrm{AL}$ would result in a refraction error of approximately $0.27 \mathrm{D}$, while the minimum detectable change in subjective refraction is $0.25 \mathrm{D}$, this difference is clinically negligible [17]. A notable advantage of the OA-2000 is its high success rate in
AL measurements. In our sample of 6465 cases, the IOLMaster did not succeed to measure AL in $4.7 \%$ of cases. Among those cases, in more than $94 \%$, a reliable $\mathrm{AL}$ measurement was achieved with the OA-2000. According to that, we can estimate a $99.7 \%$ success rate of AL reading using the OA-2000. This may reduce the number of ultrasound measurements taken, saving valuable time and lowering the inaccuracies associated with it. This superiority of the OA-2000 could favor the use of this device.

Aside from AL error, keratometry reading error is one of the most common causes of a postoperative refractive error [1, 17]. High correlation was found in the average $K$ measurements between the three devices $(\mathrm{ICC}=0.998)$. Nevertheless, there was a significant difference of $0.07 \mathrm{D}$ between the OA-2000 and the Lenstar, with the 95\% LoA range of $0.63 \mathrm{D}$. On the other hand, this difference is below the limit of clinical significance.

Compared with the IOLMaster and the Lenstar, statistically significant differences were observed for the astigmatism $X$ and $Y$ axes components values. However, the mean differences were small and the agreement was high. The results showed that the IOLMaster and Lenstar provided slightly WTR astigmatism measurements compared to the OA-2000.

We also observed good agreement (according to ICCs and LoA) in ACD, WTW, and LT measurements. As for $\mathrm{AL}$ and $K$ data, these minor, yet significant, differences seem to have little consequence on IOL calculations.

Further integration of the measurements taken by these three devices into different IOL power calculation formulas produced similar results, with minor, non-significant, differences. Comparing the absolute error of the calculated target refraction using different formulas showed similar prediction errors using the three devices with minor differences in the median absolute error $(0.00-0.03 \mathrm{D}$, depending on the formula). This range of changes is far 
Table 2 Errors in the predicted refraction using different IOL power calculation formulas and errors in the predicted residual astigmatism using Barrett's toric calculator by measuring device

\begin{tabular}{|c|c|c|c|c|}
\hline & OA-2000 & IOLMaster & Lenstar & $P$-value \\
\hline \multicolumn{5}{|l|}{ Barrett Universal II } \\
\hline Mean absolute error \pm SD & $0.25 \pm 0.19$ & $0.25 \pm 0.19$ & $0.25 \pm 0.18$ & 0.274 \\
\hline Median absolute error & 0.21 & 0.22 & 0.24 & \\
\hline [range] & {$[0.00,0.78]$} & {$[0.01,0.87]$} & {$[0.00,0.82]$} & \\
\hline Mean numeric error $\pm \mathrm{SD}$ & $-0.12 \pm 0.29$ & $-0.13 \pm 0.29$ & $-0.11 \pm 0.29$ & 0.994 \\
\hline [range] & {$[-0.78,0.67]$} & {$[-0.87,0.69]$} & {$[-0.82,0.66]$} & \\
\hline \multicolumn{5}{|l|}{ Hoffer Q } \\
\hline Mean absolute error \pm SD & $0.29 \pm 0.21$ & $0.28 \pm 0.21$ & $0.28 \pm 0.21$ & 0.151 \\
\hline Median absolute error & 0.24 & 0.24 & 0.24 & \\
\hline [range] & {$[0.00,0.82]$} & {$[0.00,1.03]$} & {$[0.00,0.93]$} & \\
\hline Mean numeric error $\pm \mathrm{SD}$ & $0.04 \pm 0.35$ & $0.02 \pm 0.35$ & $0.02 \pm 0.35$ & 0.996 \\
\hline [range] & {$[-0.82,0.73]$} & {$[-0.77,1.03]$} & {$[-0.93,0.81]$} & \\
\hline \multicolumn{5}{|l|}{ Holladay-1 } \\
\hline Mean absolute error \pm SD & $0.26 \pm 0.20$ & $0.27 \pm 0.20$ & $0.27 \pm 0.21$ & 0.951 \\
\hline Median absolute error & 0.23 & 0.24 & 0.22 & \\
\hline [range] & {$[0.00,0.85]$} & {$[0.00,0.83]$} & {$[0.01,0.94]$} & \\
\hline Mean numeric error $\pm \mathrm{SD}$ & $-0.05 \pm 0.33$ & $-0.06 \pm 0.34$ & $-0.06 \pm 0.34$ & 0.933 \\
\hline [range] & {$[-0.85,0.74]$} & {$[-0.83,0.74]$} & {$[-0.94,0.70]$} & \\
\hline \multicolumn{5}{|l|}{ SRK/T } \\
\hline Mean absolute error \pm SD & $0.27 \pm 0.21$ & $0.27 \pm 0.21$ & $0.27 \pm 0.22$ & 0.972 \\
\hline Median absolute error & 0.21 & 0.22 & 0.21 & \\
\hline [range] & {$[0.01,0.91]$} & {$[0.00,0.91]$} & {$[0.00,0.93]$} & \\
\hline Mean numeric error $\pm \mathrm{SD}$ & $-0.08 \pm 0.34$ & $-0.09 \pm 0.33$ & $-0.09 \pm 0.34$ & 0.996 \\
\hline [range] & {$[-0.91,0.83]$} & {$[-0.91,0.75]$} & {$[-0.93,0.86]$} & \\
\hline \multicolumn{5}{|l|}{ Barrett's toric calculator } \\
\hline Mean absolute error \pm SD & $0.50 \pm 0.30$ & $0.52 \pm 0.37$ & $0.46 \pm 0.33$ & 0.506 \\
\hline Median absolute error & 0.44 & 0.43 & 0.36 & \\
\hline [range] & {$[0.03,1.37]$} & {$[0.07,1.79]$} & {$[0.01,1.68]$} & \\
\hline Centroid error $(\mathrm{D}) \pm \mathrm{SD}$ at axis & $0.06 \mathrm{D} \pm 0.59$ at $13.4^{\circ}$ & $0.20 \mathrm{D} \pm 0.61$ at $14.8^{\circ}$ & $0.16 \mathrm{D} \pm 0.55$ at $21.4^{\circ}$ & $\begin{array}{l}X \text {-axis: }<0.001 \\
Y \text {-axis: } 0.011^{\mathrm{a}}\end{array}$ \\
\hline
\end{tabular}

${ }^{a} X$-axis: OA-2000 < IOLMaster, Lenstar; $Y$-Axis: OA-2000 < IOLMaster, Lenstar

The OA-2000 is manufactured by Tomey GmbH, Nürnberg, Germany

The IOLMaster-500 is manufactured by Carl Zeiss Meditec AG, Jena, Germany

The Lenstar-LS900 is manufactured by Haag-Streit AG, Koeniz, Switzerland

below the smallest subjective refraction detectable value of $0.25 \mathrm{D}$ and therefore is not clinically relevant.

Comparing the absolute astigmatism error in the subgroup of the toric IOLs, no significant difference was found between the three devices. Although the absolute residual astigmatism prediction error reflects the clinical impact of the astigmatic outcome, it does not consider the axis. Therefore, the centroid residual astigmatism prediction error, which is calculated from the $X$ and $Y$ components of the astigmatism and considers both the astigmatic magnitude and direction, has been recommended as a preferred parameter to compare astigmatic outcomes after cataract surgery $[15,18]$. In our study, the OA-2000 provided the smallest centroid error $(0.06$ vs. $0.20 \mathrm{D}$, IOLMaster, and $0.16 \mathrm{D}$, Lenstar). These results may be attributed to the 256 reference points in each measurement of the OA-2000 topography.

Our findings correlate well with previous studies. Goebels et al. [4] compared the OA-2000 biometric measurements with those of the IOLMaster and Lenstar. Similarly to us, they found that the OA-2000 measurements correlated well with the values of the two other devices, showing very small, though significant differences in mean values of $\mathrm{AL}$ and ACD and $K$. Kongsap [5] also observed similar results 


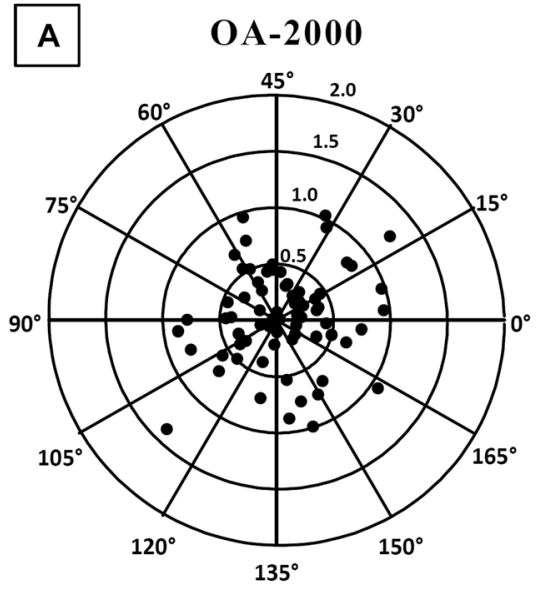

$0.06 \mathrm{D} \pm 0.59 @ 13.4^{\circ}$

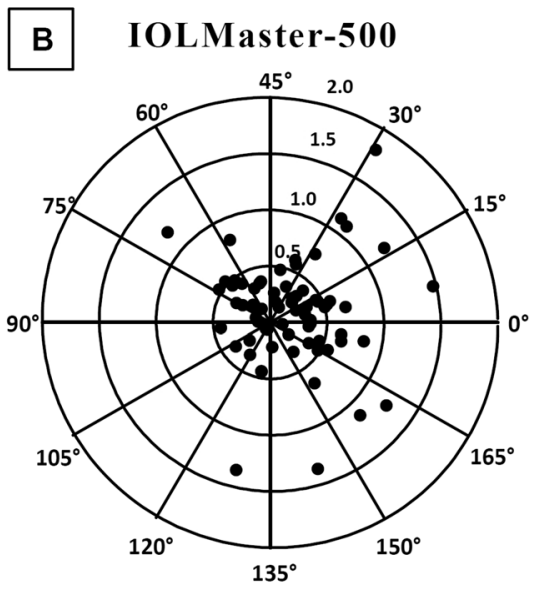

$0.20 \mathrm{D} \pm 0.61 @ 14.8^{\circ}$

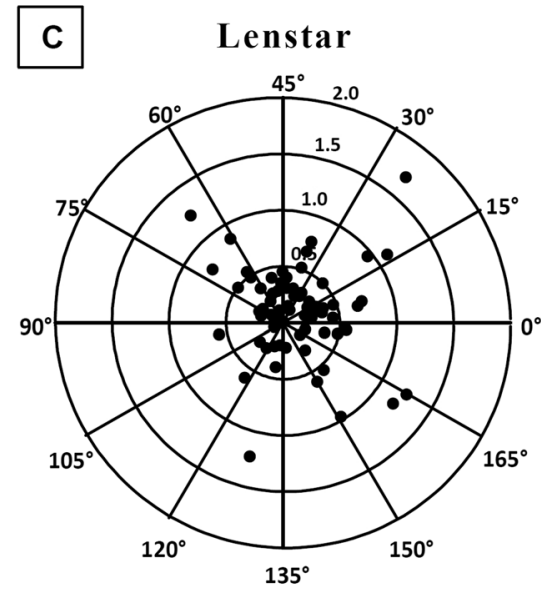

$0.16 \mathrm{D} \pm 0.55 @ 21.4^{\circ}$
Fig. 3 Double-angle plots of errors in predicted residual astigmatism with the Barrett toric calculator using a OA-2000 (Tomey $\mathrm{GmbH}$, Nagoya, Japan) measurements, b IOLMaster (Carl Zeiss Meditec,

for the AL, $K$, and ACD measurements using the OA-2000 and the IOLMaster. However, in contrast to us, he found only weak correlation of the WTW diameter. Huang et al. [6] compared the OA-2000 with the IOLMaster v5.4. They performed a Bland-Altman analysis showing narrow $95 \%$ LoA for most parameters, indicating excellent agreement for AL, $K$ values, and ACD. As for power calculation, Kongsap [5] assessed IOL power calculation using the SRK/T formula. He reported similar IOL power deriving from both instruments (mean difference of $0.32 \mathrm{D}$ ), with high correlation $(r=0.989)$. However, in contrast to our analysis, which compared the accuracy of power calculation based on the postoperative refractive results, this information was not available in this study. Recently, Savini et al. [7] evaluated IOL power calculation using the OA-2000 device for third-generation formulas. They found a median absolute error ranging between $0.33 \mathrm{D}$ (Holladay-1) and 0.35 D (SRK/T), which was greater than the median error in our study. Another difference was the finding of a lower median absolute error using the OA-2000 in comparison to the IOLMaster, which was not observed in our study. In contrast to us, they did not evaluate calculations with newer formulas such as the Barrett universal II formula and calculations for astigmatism correction with toric IOLs.

There are some limitations to our study beginning with its retrospective nature. In addition, due to the variability of IOLs in this study, we could not optimize the IOL constants, which could affect the refractive errors. Finally, this study did not compare AL measurement success rate between the OA-2000 and the Lenstar.

In conclusion, the OA-2000 biometer measurement showed excellent consistency with the IOLMaster-500 and Lenstar-LS900 measurements. Several statistical significant differences were found, but they were not clinically
Jena, Germany) measurements, and c Lenstar (Haag-Streit AG, Koeniz, Switzerland) measurements

significant, and had no influence on monofocal IOL power calculations. Regarding toric IOLs astigmatism correction, our results may imply a potential advantage using the OA-2000 device. A study on a larger number of eyes is warranted to evaluate this finding in greater depth. A notable superiority of the OA-2000 device was its higher AL measurement success rate in comparison with the IOLMaster.

\section{Summary}

\section{What was known before}

- Biometry measurements of the OA-2000 correlate well with the IOLMaster-500 and Lenstar-LS900. There are scant data available comparing the predicted refraction of various IOL power calculation formulas using the biometric data provided by these three devices.

\section{What this study adds}

- OA-2000 biometric measurements are compatible with the IOLMaster-500 and Lenstar-LS900 for power prediction using various IOL power calculation formulas. The OA-2000 device shows higher success rate in AL measurement in comparison with the IOLMaster500. The OA-2000 measurements, using the Barrett toric calculator, yielded a lower ATR centroid prediction error compared to IOLMaster-500 and Lenstar.

\section{Compliance with ethical standards}

Conflict of interest OR received partial travel reimbursement from Tomey Corporation and T.Y.R. SKYMED LTD; AL received travel 
reimbursement from Hanita Lenses; GK is a consultant of Hanita Lenses; EIA is a consultant of Hanita Lenses.

\section{References}

1. Norrby S. Sources of error in intraocular lens power calculation. J Cataract Refract Surg. 2008;34:368-76.

2. Buckhurst PJ, Wolffsohn JS, Shah S, Naroo SA, Davies LN, Berrow EJ. A new optical low coherence reflectometry device for ocular biometry in cataract patients. $\mathrm{Br} \mathrm{J}$ Ophthalmol. 2009;93:949-53.

3. Holzer MP, Mamusa M, Auffarth GU. Accuracy of a new partial coherence interferometry analyser for biometric measurements. $\mathrm{Br}$ J Ophthalmol. 2009;93:807-10.

4. Goebels S, Pattmöller M, Eppig T, Cayless A, Seitz B, Langenbucher A. Comparison of 3 biometry devices in cataract patients. $\mathrm{J}$ Cataract Refract Surg. 2015;41:2387-93.

5. Kongsap P. Comparison of a new optical biometer and a standard biometer in cataract patients. Eye Vis (Lond, Engl). 2016;3:27.

6. Huang J, Savini G, Hoffer KJ, Chen H, Lu W, Hu Q, et al. Repeatability and interobserver reproducibility of a new optical biometer based on swept-source optical coherence tomography and comparison with IOLMaster. $\mathrm{Br} \mathrm{J}$ Ophthalmol. 2017;101:493-8.

7. Savini G, Hoffer KJ, Shammas HJ, Aramberri J, Huang J, Barboni P. Accuracy of a new swept-source optical coherence tomography biometer for IOL power calculation and comparison to IOLMaster. J Refract Surg. 2017;33:690-5.
8. Hoffer KJ. The Hoffer Q formula: a comparison of theoretic and regression formulas. J Cataract Refract Surg. 1993;19:700-12.

9. Zuberbuhler B, Morrell AJ. Errata in printed Hoffer Q formula. J Cataract Refract Surg. 2007;33:2.

10. Holladay JT, Prager TC, Chandler TY, Musgrove KH, Lewis JW, Ruiz RS. A three-part system for refining intraocular lens power calculations. J Cataract Refract Surg. 1988;14:17-24.

11. Retzlaff JA, Sanders DR, Kraff MC. Development of the SRK/T intraocular lens implant power calculation formula. J Cataract Refract Surg. 1990;16:333-40.

12. Barrett GD. Intraocular lens calculation formulas for new intraocular lens implants. J Cataract Refract Surg. 1987;13:389-96.

13. Barrett GD. An improved universal theoretical formula for intraocular lens power prediction. J Cataract Refract Surg. 1993;19:713-20.

14. Barrett GD. Calculating astigmatic correction. Presented at the XXXIV Congress of the European Society of Cataract and Refractive Surgeons, September 2016, Copenhagen, Denmark.

15. Holladay JT, Moran JR, Kezirian GM. Analysis of aggregate surgically induced refractive change, prediction error, and intraocular astigmatism. J Cataract Refract Surg. 2001;27:61-79.

16. Bland JM, Altman DG. Statistical methods for assessing agreement between two methods of clinical measurement. Lancet. 1986;1:307-10.

17. Olsen T. Calculation of intraocular lens power: a review. Acta Ophthalmol Scand. 2007;85:472-85.

18. Alpins NA, Goggin M. Practical astigmatism analysis for refractive outcomes in cataract and refractive surgery. Surv Ophthalmol. 2004;49:109-22. 